\title{
PrefEREnCE Intensity REPRESENTATION: Strategic Overstating in Large ELECTIONS*
}

\author{
MATÍAS NÚÑ̃EZ \\ AND \\ JEAN-FRANÇOIS LASLIER ${ }^{\ddagger}$
}

SEPTEMBER 2012

\begin{abstract}
If voters vote strategically, is it useful to offer them the possibility of expressing nuanced opinions, or would they always overstate the intensity of their preferences? For additive voting rules, say that a ballot is extremal if it is neither abstention-like nor can be expressed as a mixture of the available ballots. We give a sufficient condition for strategic equivalence: if two rules share the same set of extremal ballots (up to an homothetic transformation), they are strategically equivalent in large elections. This condition is also necessary for the strategic equivalence of positional rules. These results do not hold for small electorates.
\end{abstract}

KEYWORDS: Strategic voting, voting equilibria.

JEL Classification Numbers: D70; D72.

${ }^{*}$ This work has benefited from useful discussions with Francis Bloch, Seok-ju Cho, Francesco De Sinopoli, Santiago Oliveros and Eduardo Perez-Richet. This work has also benefited from comments of conference and seminar participants in the American Political Science Association Meeting 2011 - Seattle, OECD - New Directions in Welfare Conference 2011, the Workshop Advances on Approval Voting 2010, Université Paris II, THEMA - Université de Cergy-Pontoise and Ecole Polytechnique. We would also like to thank the editor John Duggan and one referee for very useful suggestions that substantially upgraded the quality of this work.

${ }^{\dagger}$ Corresponding Author. CNRS and Université de Cergy-Pontoise, THEMA, F-95000 CergyPontoise, France. Email: matias.nunez@u-cergy.fr.

${ }^{\ddagger}$ CNRS and Department of Economics, Ecole Polytechnique, France. 


\section{Introduction}

One of the reasons advocated for extending the set of ballots available to the voters is to allow them to represent more accurately the intensity of their preferences for the various candidates. Consider for instance Evaluative Voting $(E V)$. Under $E V$, the voter evaluates each candidate independently on the same numerical scale, the grades are added and the candidate with the largest total is elected. Baujard and Igersheim (2010) [2] and Baujard et al. (2012) [1] report on field work on $E V$ with various scales, and observe that voters often say that they appreciate the possibility of voicing their opinions more finely than what a uni-nominal vote allows.

Evaluative Voting is an extension of Approval Voting $(A V)$, which can be considered as EV with the simple scale $\{0,1\}$. And $A V$ is itself an extension of Plurality Voting $^{1}(P V)$, in the sense that a voting rule $V^{\prime}$ is an extension of a voting rule $V$ if all the ballots available under $V$ are available under $V^{\prime}$.

But does extending the set of ballots available under a given voting rule modify the set of voting equilibria? For instance, Dhillon and Mertens (1999) [9] study the "Relative Utilitarianism" mechanism, according to which voters grade alternatives and the voter's grades are summed after re-scaling to the interval $[0,1]$. They write: "Except possibly with very small sets of voters, voters will clearly find that, for their votes to have a maximal effect, they should assign either 0 or 1 to every alternative. Hence the corresponding direct mechanism seems to be 'approval voting'." Indeed, assuming strategic voting, one might suspect that the set of voting equilibria should often not be altered by such an extension.

We shall verify this intuition for large electorates. However, it need not be always the case that extending the set of ballots has no strategic implications. For instance, $A V$ is an extension of $P V$ and, as shown by Myerson and Weber (1993) [24], $A V$ improves the aggregation of preferences when compared with $P V$ in the noteworthy divided majority situation.

We here focus on additive voting rules, in which a ballot is a list of points that the

\footnotetext{
${ }^{1} \mathrm{EV}$ is obviously related to Utilitarianism; see Karni (1998) [13], Dhillon and Mertens (1999) [9], Segal (2000) [31], d'Aspremont and Gevers (2002) [7] and Gaertner and Xu (2012) [10] who called it Range Voting, for axiomatic analyses. $A V$ is often advocated since it emerged in the literature in the mid 70s; see Laslier and Sanver (2010) [17] for a detailed account. In an election held under $P V$, a voter is allowed to give at most one point to at most one candidate and the candidate with the most votes wins the election. The most common rule for direct presidential elections is Plurality with a Runoff (Blais (1997) [3]), but we here restrict attention to one-round voting systems.
} 
voter is affording to the candidates, and where points for each candidate are simply added. (A formal definition of this family of voting rules is provided in the next section.) We analyze the issue in the framework of strategic voting, that is assuming that voters strategically cast their votes in order to maximize their (expected) utility. We study equilibria and consider that two voting rules are strategically equivalent if they have the same equilibrium outcomes.

Strategic incentives in large and small electorates may differ and have been modeled in different ways. To study small electorates, we use a standard refinement of Nash equilibrium (perfectness) and provide an example that shows that voters need not overstate at equilibrium.

To tackle the problem on large elections, we focus on one of the first and simplest models in this direction, proposed by Myerson and Weber (1993) [24] $]^{2}$. In this model, for any pair of candidates, the voter considers that there is a positive probability that her vote is pivotal on this pair, but some of these probabilities are vanishingly small compared to others. We first define the notion of strategically equivalent voting rules. Two equilibria of elections held under two different rules are equivalent if and only if the winning candidates are the same ones and voters' strategies can be justified by the same sequence of pivot probabilities. Two voting rules are strategically equivalent if and only if to each equilibrium for one rule corresponds an equivalent equilibrium for the other rule.

We then derive necessary and sufficient conditions for the strategic equivalence of voting rules. The sufficient condition is simple. Say that a ballot is extremal if it is not abstention-like (the ballot does not treat all candidates alike) and it cannot be expressed as a mixture of other available ballots. Notice that "casting an extremal ballot" does not here mean voting for a candidate at some extreme point of the political spectrum, it means voicing a (maybe moderate) political opinion in the strongest possible way.

If two voting rules offer the same set of extremal ballots, up to a homothetic transformation, then they are strategically equivalent. The use of this sufficient condition is fairly straightforward, implying several interesting consequences.

The first consequence is that Approval Voting and Evaluative Voting are strategically equivalent. The second consequence concerns a different family of voting rules. In an election held under Cumulative Voting $(C V)$, another natural extension of Plurality Voting, a voter is endowed with a finite number of points, and he is al-

\footnotetext{
${ }^{2}$ Other models of large electorates have been proposed: Palfrey (1989) [28], Laslier (2000) [14], Myerson (2000) [22] and McKelvey and Patty (2006) [18].
} 
lowed to distribute them freely between the different candidates. Different authors ${ }^{3}$ have discussed such a method. With such a voting rule, voters have the possibility of choosing an extremal ballot: that is to give the highest possible amount of points to only one of the candidates. We prove that this is indeed the case in equilibrium, implying that $P V$ and $C V$ are strategically equivalent. We hence prove that for both $P V$ and $A V$, there exist extensions that do not modify the set of voting equilibria.

A related question is to know whether extending a voting rule, and hence giving more flexibility to the voters, necessarily leads to a better preference aggregation. Such is the case with $P V$ and $A V$ in the examples of Myerson and Weber (1993) [24]. Call robust the voting rules whose set of equilibria remains unaltered by any finite extension. As we will see, provided that the maximal score that can be attached to a candidate is bounded, for any voting rule $U$, there must exist an extension $V$ such that the set of extremal ballots of $V$ coincide with the set of extremal ballots of $E V$ with the maximum number of points. Due to the sufficient condition, $V$ and $E V$ are strategically equivalent. Therefore, giving more flexibility might modify the set of equilibria, however, the limit of such extensions is strategically equivalent to $E V$ with the maximum number of points and therefore to $A V$. Therefore, the more flexibility a voting rule allows to voters, the more it becomes similar, from a strategic point of view, to Approval Voting.

As far as the necessary condition is concerned, we prove that the existence of a homothetic transformation is necessary for strategic equivalence if we restrain the allowed voting rules to be rank, or positional, scoring rules. A rank scoring rule (as axiomatized by Young (1975) [34]) is characterized by a vector of weights to be assigned to each of the candidates, non-abstention ballots being all the permutations of this vector. The candidate(s) with the highest number of votes is (are) declared the winner(s) of the election. For instance, $P V$, Borda Count and Negative Voting are rank scoring rules whereas $A V$ is considered as a non-rank scoring rule, since such a permutation does not exist. The reason of this simple necessary condition is the structure of positional rules, any extremal ballot being a permutation of the same vector. In full generality, that is without imposing some further structure to the rules, a tractable necessary condition seems to be hard to obtain.

The described equivalence between voting equilibria is valid along the lines of the theory of large elections proposed by Myerson and Weber (1993) [24]. Small

\footnotetext{
${ }^{3}$ See Sawyer and McRae (1962) [30], Brams (1975) [5], Nitzan (1985) [25], Cox (1990) [6] and Gerber et. al (1998) [11].
} 
elections (that is elections with few voters) raise new questions. For instance, the information available to voters might be much more detailed in a small election than in a mass election, implying that a theory such as Myerson and Weber's one is of scant interest for small electorates. In order to investigate whether the previous claims still hold in environments with few voters, we discuss a voting situation in the case of Evaluative Voting.

In the example that we study, the strategy combination is a mixed-strategy equilibrium in which the unique pure strategy best response for a voter is not extremal. Indeed, one of the voters of the election mixes between his undominated strategies making uncertain the final electoral outcome for the rest of the voters. The "mixing" removes the weak preference for overstatements. Furthermore, the strategy profile is a perfect equilibrium à la Selten (1975) [32]. This situation proves that the lack of overstatement can be a best response even in equilibria that satisfy different equilibria refinements. The refinement (trembling-hand perfection) used in this work is among the most classical ways of obtaining equilibria as a limit of games with small uncertainty (i.e. perturbed games). However, it should not be too difficult to generalize the results to settings in which the uncertainty comes from other sources. For instance, Bayesian games with some uncertainty about voters' types or common values' settings with imperfect information about the true state of nature are good candidates for models in which extremal voting is not always a best response for a strategic voter.

This paper is organized as follows. Section 2 presents the basics of the model. Section 3 to 5 are devoted to large elections: Section 3 describes the equilibrium concept, Section 4 states the sufficient and necessary conditions for strategic equivalence, Section 5 presents the strategic equivalence between the above-mentioned voting rules and contains the results on the robustness of a voting rule. Section 6 presents the results concerning the environments with few voters, and Section 7 provides some concluding comments.

\section{The setting}

The finite set of voters is denoted by $\mathcal{N}=\{1, \ldots, n\}$. Each voter has a type $t$ that determines his preferences over the set of candidates $\mathcal{K}=\left\{c_{1}, c_{2}, \ldots, c_{k}\right\}$. The preferences of a voter with type $t$ (a $t$-voter) is defined by $u_{t}=\left(u_{t}\left(c_{i}\right)\right)_{c_{i} \in \mathcal{K}}$, in which $u_{t}\left(c_{i}\right)$ denotes the utility a $t$-voter gets if candidate $c_{i}$ wins the election. Voters' utilities are assumed not to be constant across all candidates. All types $t$ belong to 
a finite set of types $\mathcal{T}$. The distribution of types is denoted by $r=(r(t))_{t \in \mathcal{T}}$ with $\sum_{t} r(t)=1$, in other words, $r(t)$ represents the share of $t$-voters.

In this work, we stick to the comparison of additive rules: a ballot is a vector $b=\left(b_{1}, b_{2}, \ldots, b_{k}\right)$ where $b_{i}$ is a positive integer that denotes the number of points given to candidate $c_{i}$, to be added to elect the candidate with the largest score. We assume that the number of points is bounded, i.e. there exists some positive real number $M$ such that $b_{i} \leq M$ for every $i$. Each voter must choose a ballot $b$ from a finite set of possible ballots denoted by $\mathcal{B}$.

Hence, a voting game is characterized by $(\mathcal{N}, \mathcal{T}, \mathcal{K}, \mathcal{B}, r)$. Among additive voting rules, we will pay special attention to Evaluative, Cumulative, Approval, and Positional rules.

Under Evaluative Voting with $m$ points, a voter can assign up to $m$ points to each candidate. Hence:

$$
b \text { is an } E V^{m} \text { ballot if } \forall c_{j} \in \mathcal{K}, b_{j} \in\{0,1, \ldots, m\} \text {. }
$$

Similarly, an Approval Voting $(A V)$ ballot consists of a vector that lists whether each candidate has been approved or not: for each $c_{j} \in \mathcal{K}, b_{j} \in\{0,1\}$. Hence, $A V$ is a particular case of $E V^{m}$ with $m=1$.

In an election held under Cumulative Voting with $m$ points, a voter can assign up to $m$ points to each candidate, with the restriction that the sum of the points he assigns to the candidates is at most $m$ :

$$
b \text { is an } C V^{m} \text { ballot if } \forall c_{j} \in \mathcal{K}, b_{j} \in\{0,1, \ldots, m\} \text {, and } \sum_{c_{j} \in \mathcal{K}} b_{j} \leq m \text {. }
$$

In an election held under Plurality Voting $(P V)$ voters can abstain or give one point to at most one candidate. Hence, $P V$ is a particular case of $C V^{m}$ with $m=1$.

Formally, we can write, with obvious notation:

$$
\begin{aligned}
& \mathcal{B}_{E V^{m}}=\{0,1, \ldots, m\}^{\mathcal{K}}, \mathcal{B}_{A V}=\mathcal{B}_{E V^{1}} \\
& \mathcal{B}_{C V^{m}}=\left\{b \in\{0,1, \ldots, m\}^{\mathcal{K}}: \sum_{c_{j} \in \mathcal{K}} b_{j} \leq m\right\}, \mathcal{B}_{P V}=\mathcal{B}_{C V^{1}} .
\end{aligned}
$$

Following Myerson (1999) [21], a rank scoring rule (a positional voting rule) $U$ in a $k$-candidates election is characterized by some list of numbers $b=\left(b_{1}, b_{2}, \ldots, b_{k}\right)$ such that $b_{1} \geq b_{2} \geq \ldots \geq b_{k}=0$, and the ballots are all the permutations of $b$. For instance, Plurality voting is such that $b_{1}=1$ and $b_{j}=0$ for any $j \neq 1$. Similarly, 
the Borda rule satisfies $b_{j}=\frac{k-j}{k-1}$. Negative voting belongs to this family as $b_{k}=0$ and $b_{j}=1$ for any $j \neq k$. Such rules play an important role in social choice theory and were early characterized by Smith (1973) [33] and Young (1975) [34].

Note that both $E V^{m}$ for any $m$ and $C V^{m}$ for $m>1$ are non-rank scoring rules as it is not be the case that all ballots in the ballot set are permutations of the same vector. Central to this paper is the following concept:

Definition 1. A voting rule $V$ is an extension of the voting rule $V^{\prime}$ if all ballots in $V^{\prime}$ are available in $V$, i.e.

$$
\mathcal{B}_{V^{\prime}} \subset \mathcal{B}_{V}
$$

For instance, given a finite $m, E V^{m}$ is an extension of $C V^{m}$ as $\mathcal{B}_{C V^{m}} \subset \mathcal{B}_{E V^{m}}$. Similarly, Approval Voting is an extension of Plurality Voting.

\section{$3 \quad$ Large Elections}

We assume that each voter maximizes his expected utility to determine which ballot in the set $\mathcal{B}$ he will cast. In this model, his vote has an impact in his payoff if it changes the winner of the election. Therefore, a voter needs to estimate the probability of these situations: the pivot events. We say that two candidates are tied if their vote totals are equal. Furthermore, let $H$ denote the set of all unordered pairs of candidates, we denote a pair $\left\{c_{i}, c_{j}\right\}$ in $H$ as $i j$ with $i j=j i$.

For each pair of candidates $c_{i}$ and $c_{j}$, the $i j$-pivot probability $p_{i j}$ is the probability of the outcome perceived by the voters that candidates $c_{i}$ and $c_{j}$ will be tied for first place in the election. A voter perceives that the probability that he will change the winner of the election from candidate $c_{i}$ to candidate $c_{j}$ by casting ballot $b$ with $b_{i} \geq b_{j}$ to be linearly proportional to $b_{i}-b_{j}$, and that the constant of proportionality (the $i j$-pivot probability) is the same for the perceived chance of changing the winner from $j$ to $i$ if $b_{j} \geq b_{i}{ }^{4}$.

\footnotetext{
${ }^{4}$ This is roughly equivalent to assume that the probability of candidates $c_{i}$ and $c_{j}$ being tied for first place is the same as the probability of candidate $c_{i}$ being in first place one point ahead of candidate $c_{j}$ (and both candidates above the rest of the candidates), which is in turn the same one as the probability of candidate $c_{j}$ being in first place one vote ahead of candidate $c_{i}$. Myerson and Weber (1993) [24] justify this assumption by arguing that it seems reasonable when the electorate is large enough. This is not verified in Poisson games, a formal model of large elections in which the pivot probabilities are derived endogenously from the structure of the game.
} 
A vector listing the pivot probabilities for all pairs of candidates is denoted by $p=\left(p_{i j}\right)_{i j \in H}$. This vector $p$ is assumed to be identical and common knowledge for all voters in the election. A voter with $i j$-pivot probability $p_{i j}$ anticipates that submitting the ballot $b$ can change the winner of the election from candidate $c_{j}$ to candidate $c_{i}$ to be $p_{i j} \max \left\{b_{i}-b_{j}, 0\right\}$.

A (voting) strategy is a probability distribution $\sigma$ over the set $\mathcal{B}$ that summarizes the voting behavior of voters of each type. For any ballot $b$ and any type $t, \sigma(b \mid t)$ is the probability that a $t$-voter casts ballot $b$. Let $\sigma(\cdot \mid-t)$ stands for the strategy profile of the voters with types different from $t$. The expected utility gain from casting ballot $b$ equals the expected utility of casting ballot $b$ minus the expected utility of abstaining. Focusing on utility gains simplifies notation. The expected utility gain of a $t$-voter when he plays the strategy $\sigma(\cdot \mid t)$ equals $E_{t}[\sigma(\cdot \mid t) \mid p]$ where $p$ is the common vector of pivot probabilities. Slightly abusing notation, we let $E_{t}[b \mid p]$ denote the expected utility gain of a $t$-voter from casting ballot $b$, that is he plays the pure strategy $b$ with:

$$
E_{t}[b \mid p]=\sum_{i j \in H}\left(b_{i}-b_{j}\right) \cdot p_{i j} \cdot\left[u_{t}\left(c_{i}\right)-u_{t}\left(c_{j}\right)\right] .
$$

Given the strategy combination $\sigma$, the share of the electorate who cast ballot $b$ is denoted by

$$
\tau(b)=\sum_{t \in \mathcal{T}} r(t) \sigma(b \mid t),
$$

hence, the expected score of candidate $c_{i}$ is

$$
S\left(c_{i}\right)=\sum_{b \in \mathcal{B}} b_{i} \tau(b)
$$

The score vector $S=\left(S\left(c_{i}\right)\right)_{c_{i} \in \mathcal{K}}$ describes the expected score of each candidate in the election. The set of likely winners $W(\mathcal{K})$ of the election contains the candidates whose expected score $S\left(c_{i}\right)$ is maximal given the strategy $\sigma$.

Myerson and Weber (1993) [24] assume that voters expect candidates with lower expected scores to be less likely serious contenders for first place than candidates with higher expected scores. In other words, if the expected score for some candidate $c_{i}$ is strictly higher than the expected score for some candidate $c_{j}$, then the voters would perceive that candidate $c_{i}$ 's being tied with any third candidate $c_{l}$ is much more likely than candidate $c_{j}$ 's being tied for first place with candidate $c_{l}$.

Definition 2. Given a voting strategy $\sigma$ and any $0<\varepsilon<1$, a pivot probability vector $p$ satisfies the ordering condition for $\varepsilon$ given $\sigma$ if, for every three distinct 
candidates $c_{i}, c_{j}$ and $c_{l}$ :

$$
S\left(c_{i}\right)>S\left(c_{j}\right) \Longrightarrow p_{j l} \leq \varepsilon p_{i l} .
$$

Besides, Myerson and Weber (1993) [24] assume that the probability of three (or more) candidates being tied for first place is infinitesimal in comparison to the probability of a two-candidate tie.

Given a pivot probability vector $p$, the set of pure best replies of $t$-voters is as follows:

$$
\operatorname{BR}_{t}(p)=\left\{b \in \mathcal{B} \mid b \in \arg \max _{d \in \mathcal{B}} E_{t}[d \mid p]\right\}
$$

Given a strategy combination $\sigma$, the support of $\sigma$ for $t$-voters denotes the set of pure strategies (ballots) played with positive probability by $t$-voters according to $\sigma$ :

$$
\operatorname{Supp}_{t}(\sigma)=\{b \in \mathcal{B} \mid \sigma(b \mid t)>0\} .
$$

Definition 3. The strategy $\sigma$ is a voting equilibrium of the game if and only if, for every positive number $\varepsilon$, there exists a vector $p^{\varepsilon}$ of positive pivot probabilities that satisfies, given $\sigma$, the ordering condition and such that, for each ballot $b$ and for each type $t$,

$$
b \in \operatorname{Supp}_{t}(\sigma) \Longrightarrow b \in B R_{t}\left(p^{\varepsilon}\right) .
$$

It should be stressed that, in this definition, the pivot probabilities $p_{i j}^{\varepsilon}$ are supposed to be the same when the voter contemplates casting one ballot or the other. This point will play an important role in the next section. It is justified when the number of voters is large for, in that case, the voter cannot change with his single vote the order of magnitude of these probabilities. It can be shown that the set of equilibria is non-empty ${ }^{5}$.

Dividing the positive vector $p^{\varepsilon}$ by the sum of its components, one obtains a probability distribution $q^{\varepsilon}=\left(q_{i j}^{\varepsilon}\right)_{i j \in H}$ over the set $H$ of pairs of candidates. Note that $q^{\varepsilon}$ still satisfies the ordering condition. The component $q_{i j}^{\varepsilon}$ represents the conditional probability of a pivot between candidates $c_{i}$ and $c_{j}$ in the event of a pivot between any two candidates in the election. Myerson and Weber (1993) [24] remark that, in an equilibrium, $q_{i j}^{\varepsilon}$ can have a strictly positive limit when $\varepsilon$ tends to 0 only if one of these conditions holds: either both $c_{i}$ and $c_{j}$ are the front-runners of the election or

\footnotetext{
${ }^{5}$ See Theorem 1, page 105 in Myerson and Weber (1993) [24].
} 
one of these two candidates is the front-runner and the other has the second highest expected score.

Finally, an important concept in our model should be defined: the equivalence between equilibria under different voting rules.

Definition 4 (Equivalence of Equilibria). An equilibrium $\sigma_{U}$ of an election held under a voting rule $U$ is equivalent to an equilibrium $\sigma_{V}$ of the same election held under $V$ if and only if they are justified by the same sequence of pivot probability vectors and the winning set is identical.

Definition 5. Two voting rules are strategically equivalent if and only if their set of voting equilibria are equivalent, that is if for any equilibrium of the election held under one rule there exists a equivalent equilibrium under the other.

A possible winner is a candidate who wins the election in equilibrium with positive probability. The set of possible winners of an election held under the voting rule $V$ is denoted:

$W_{V}=\left\{c_{i} \in \mathcal{K} \mid\right.$ There exists an equilibrium $\sigma$ in which $S\left(c_{i}\right)$ is maximal $\}$

If two voting rules are strategically equivalent, then they have the same set of possible winners. However, the converse needs not be true; for instance, the rankings of other candidates may differ. It is noteworthy that the definition of strategic equivalence used is rather demanding. It requires more than the set of possible winners being the same under two voting rules. This demanding definition reinforces our results as we show that this strong version of equivalence holds in the Myerson-Weber setting.

\section{Strategic equivalence}

We now introduce some categories of ballots that will be useful throughout.

An abstention ballot is a ballot with all the coordinates alike; the set of such ballots is denoted by $\operatorname{Abs}(\mathcal{B})$.

An interior ballot $b$ is a ballot which is not an abstention ballot and that can be expressed as a strict convex combination of other ballots in $\mathcal{B}$, i.e. there exist ballots

$$
b^{1}, b^{2}, \ldots, b^{m} \in \mathcal{B} \text { with } b=\sum_{i} \alpha_{i} b^{i} \text { with } \alpha_{i} \in(0,1) \text { and } \sum_{i} \alpha_{i}=1 .
$$


An extremal ballot is a ballot which is neither an interior nor an abstention ballot. Given the set of ballots $\mathcal{B}$, the set of interior and extremal ballots are respectively denoted by $\operatorname{Int}(\mathcal{B})$ and $\operatorname{Ext}(\mathcal{B})$ with,

$$
\operatorname{Ext}(\mathcal{B})=\mathcal{B} \backslash(\operatorname{Int}(\mathcal{B}) \cup \operatorname{Abs}(\mathcal{B}))
$$

Letting $\Delta(\mathcal{B})$ the set of convex combinations of ballots in $\mathcal{B}$, the extremal ballots correspond to the extreme points of $\Delta(\mathcal{B})$ minus any abstention ballots.

We need to make the following technical assumption concerning the ballot set: there exists for any type of voters a ballot in the set of ballots $\mathcal{B}$ that delivers a positive expected utility to this type.

Regular Ballot Sets: A ballot set $\mathcal{B}$ is regular if for, for every type $t$ and for every positive pivot probability vector $p^{\varepsilon}$, there exists a ballot $b^{*} \in \mathcal{B}$ that ensures a positive utility gain to $t$-voters: $E_{t}\left[b^{*} \mid p^{\varepsilon}\right]>0$.

Assuming regular ballot sets is not too restrictive. For instance, it suffices to see that a sincere ballot under a rank scoring rule (and also under $C V^{m}$ and under $E V^{m}$ ) gives a strictly positive expected utility to voters. Recall that, by assumption, voters' utilities are not constant across candidates. Assume that some voter's preferences are as follows $u_{t}\left(c_{1}\right)>u_{t}\left(c_{2}\right)>\ldots>u_{t}\left(c_{k}\right)$. Voting sincerely implies to vote $b=$ $\left(b_{1}, b_{2}, \ldots, b_{k-1}, 0\right)$ with $b_{i} \geq b_{i+1}$. By $(1), E_{t}\left[b \mid p^{\varepsilon}\right]=\sum_{i j \in H}\left(b_{i}-b_{j}\right) p_{i j}^{\varepsilon}\left[u_{t}\left(c_{i}\right)-u_{t}\left(c_{j}\right)\right]$. As $\left(b_{i}-b_{j}\right) \geq 0$ with $i<j$, with at least one inequality being strict, then $E_{t}\left[b \mid p^{\varepsilon}\right]>0$ as wanted. We assume throughout that the ballot set $\mathcal{B}$ is regular.

Lemma 1. Neither an abstention ballot nor a ballot which is a convex combination of at least one abstention ballot are cast with positive probability in equilibrium.

Proof. We let $a^{x}$ denote an abstention ballot with $a^{x}=(x, \ldots, x)$ for some positive $x$. For any $\varepsilon>0$, by (1), $E_{t}\left[a^{x} \mid p^{\varepsilon}\right]=0$ for any $p^{\varepsilon}$.

The regularity of the ballot set directly implies that any strategy combination $\sigma$ in which an abstention ballot is cast with positive probability is not an equilibrium. Indeed, as the ballot set is regular, there exists a ballot $b^{*} \in \mathcal{B}$ such that $E_{t}\left[b^{*} \mid p^{\varepsilon}\right]>$ $0=E_{t}\left[a^{x} \mid p^{\varepsilon}\right]$ for any $p^{\varepsilon}$. Therefore, $a^{x} \notin \operatorname{BR}_{t}\left(p^{\varepsilon}\right)$ which implies that $a^{x} \notin \operatorname{Supp}_{t}(\sigma)$ in any equilibrium $\sigma$.

It remains to be proven that a ballot which is a convex combination of at least one abstention ballot is not cast with positive probability in equilibrium. Let $c$ be such a ballot with $c=\alpha_{x} a^{x}+\sum_{i \in \mathcal{D}} \alpha_{i} d^{i}$. Ballot $c$ is the convex combination the abstention ballot $a^{x}$ and of ballots $d^{i}$ in some subset $\mathcal{D} \subseteq \mathcal{B}$ with $\sum_{i \in \mathcal{D} \cup\{x\}} \alpha_{i}=1$ with $\alpha_{i} \geq 0$ 
for any $i \in \mathcal{D} \cup\{x\}$. Therefore, for any $\varepsilon>0, E_{t}\left[c \mid p^{\varepsilon}\right]=\sum_{i \in \mathcal{D}} \alpha_{i} E_{t}\left[d^{i} \mid p^{\varepsilon}\right]$ as $E_{t}\left[a^{x} \mid p^{\varepsilon}\right]=0$. Suppose that there exists an equilibrium $\sigma$ with $c \in \operatorname{Supp}_{t}(\sigma)$.

Suppose first that $E_{t}\left[c \mid p^{\varepsilon}\right] \leq 0$ with respect to $\sigma$. By the regularity of the ballot set, there exists a ballot $b^{*} \in \mathcal{B}$ such that $E_{t}\left[b^{*} \mid p^{\varepsilon}\right]>0$. But then $E_{t}\left[b^{*} \mid p^{\varepsilon}\right]>0 \geq$ $E_{t}\left[c \mid p^{\varepsilon}\right]$, so that $c \notin \mathrm{BR}_{t}\left(p^{\varepsilon}\right)$. Therefore, $\sigma$ is not an equilibrium.

Suppose now that $E_{t}\left[c \mid p^{\varepsilon}\right]>0$ with respect to $\sigma$. By definition, there exists a ballot $b^{*} \in \mathcal{B}$ such that $E_{t}\left[b^{*} \mid p^{\varepsilon}\right]>0$ for any $p^{\varepsilon}$. Let $\hat{\sigma}(\cdot \mid t)$ stand for the mixed strategy of $t$-voters such that $\hat{\sigma}\left(b^{*} \mid t\right)=\alpha_{x}$ and for any $d^{i} \in \mathcal{D}, \hat{\sigma}\left(d^{i} \mid t\right)=\alpha_{i}$. Therefore, $\hat{\sigma}$ is a well-defined mixed strategy with

$$
\begin{aligned}
E_{t}\left[\hat{\sigma}(\cdot \mid t) \mid p^{\varepsilon}\right] & =\alpha_{x} E_{t}\left[b^{*} \mid p^{\varepsilon}\right]+\sum_{i \in \mathcal{D}} \alpha_{i} E_{t}\left[d^{i} \mid p^{\varepsilon}\right] \\
& >\alpha_{x} E_{t}\left[a^{x} \mid p^{\varepsilon}\right]+\sum_{i \in \mathcal{D}} \alpha_{i} E_{t}\left[d^{i} \mid p\right]=E_{t}\left[c \mid p^{\varepsilon}\right] .
\end{aligned}
$$

Therefore, as $E_{t}\left[\hat{\sigma}(\cdot \mid t) \mid p^{\varepsilon}\right]>E_{t}\left[c \mid p^{\varepsilon}\right]$, then $c \notin \mathrm{BR}_{t}\left(p^{\varepsilon}\right)$. Therefore, we can conclude that $\sigma$ is not an equilibrium.

\section{Remark 1 .}

The definition of an interior ballot implies that if a ballot $c$ is interior then it is the strict convex combination of extremal and abstention ballots:

$c=\sum_{b \in \mathcal{B}^{\prime}} \alpha_{b} \cdot b$ for some $\mathcal{B}^{\prime}$ with $\mathcal{B}^{\prime} \subset \operatorname{Ext}(\mathcal{B}) \cup \operatorname{Abs}(\mathcal{B})$ with $\alpha_{b} \in(0,1)$ and $\sum_{b \in \mathcal{B}^{\prime}} \alpha_{b}=1$.

\section{Remark 2.}

The set $\operatorname{Ext}(\mathcal{B})$ of extremal ballots is non-empty for any non-constant voting rule. To see this suppose that $\operatorname{Ext}(\mathcal{B})=\emptyset$ for some voting rule with ballot set $\mathcal{B}$. By definition, $\mathcal{B}=\operatorname{Ext}(\mathcal{B}) \cup \operatorname{Int}(\mathcal{B}) \cup \operatorname{Abs}(\mathcal{B})$. As $\mathcal{B}$ is finite, not every ballot can be expressed by a convex combination of other ballots in $\mathcal{B}$, so that $\mathcal{B} \neq \operatorname{Int}(\mathcal{B})$. As we have assumed that $\operatorname{Ext}(\mathcal{B})=\emptyset$, we must have that $\operatorname{Abs}(\mathcal{B}) \neq \emptyset$, i.e. every ballot which is not interior is an abstention ballot. Hence, every ballot of such a rule is an abstention ballot as any interior ballot is a strict convex combination of other ballots. Thus, such a voting rule can be labeled as constant as it elects for any vote distribution the whole set of candidates.

\section{Example 1.}


Consider a three-candidates election held under $C V^{m}$ with $m=2$. Each voter is endowed with at most two points that can be freely distributed among the different candidates. The set of allowed ballots $\mathcal{B}_{C V^{m}}$ is:

$$
\begin{aligned}
& \mathcal{B}_{C V^{m}}=\{(0,0,0),(2,0,0),(0,2,0),(0,0,2), \\
&(1,1,0),(1,0,1),(0,1,1),(1,0,0),(0,1,0),(0,0,1)\} .
\end{aligned}
$$

The interior ballots are:

$$
\operatorname{Int}\left(\mathcal{B}_{C V^{m}}\right)=\{(1,1,0),(1,0,1),(0,1,1),(1,0,0),(0,1,0),(0,0,1)\}
$$

(To see this, it is enough to write that for instance the interior ballot $(1,1,0)$ equals the convex combination $1 / 2(2,0,0)+1 / 2(0,2,0)$.) There is a unique abstention ballot:

$$
\operatorname{Abs}\left(\mathcal{B}_{C V^{m}}\right)=\{(0,0,0)\} .
$$

Finally, the set of extremal ballots is:

$$
\operatorname{Ext}\left(\mathcal{B}_{C V^{m}}\right)=\{(2,0,0),(0,2,0),(0,0,2)\}
$$

\subsection{Extremal Voting}

By Lemma 1, an abstention ballot is not cast with positive probability in equilibrium, so that there are two types of equilibria: interior and extremal. We refer to an interior equilibrium whenever an interior ballot is included in the support of a voter's strategy. Formally, the equilibrium $\sigma$ is interior if there exists $t \in \mathcal{T}$ such that $\operatorname{Int}(\mathcal{B}) \cap \operatorname{Supp}_{t}(\sigma) \neq \emptyset$. On the contrary, an extremal equilibrium is an equilibrium in which the support of every voter's strategy uniquely includes extremal ballots.

The following Proposition proves that for any interior equilibrium, there exists an equivalent extremal one. The logic of the proof is as follows. We start with an interior equilibrium in which at least one type of voters cast an interior ballot. We then build an equivalent equilibrium in which the support of this type of voters only includes overstating ballots. As the construction does not depend on the initial interior equilibrium, one can iteratively apply this construction until no type of voters casts an interior ballot. It hence follows that for any interior equilibrium, there must exist an equivalent equilibrium in which the support of every voter uniquely includes extremal ballots, an extremal equilibrium. 
Proposition 1. [Strategic Overstating] Let $U$ be a voting rule. For any interior equilibrium, there exists an equivalent extremal equilibrium.

Proof. Let $\sigma=(\sigma(\cdot \mid t), \sigma(\cdot \mid-t))$ be an interior equilibrium such that the $t$-voter's strategy satisfies $\sigma(c \mid t)=1$ with $c$ being an interior ballot. Similar arguments can be used to extend the proof whenever $\sigma$ involves that $t$-voters play in mixed strategies.

As $c$ is an interior ballot, we can write $c=\sum_{i \in \mathcal{D}} \alpha_{i} d^{i}$, that is $c$ is a convex combination of ballots $d^{i}$ in some subset $\mathcal{D} \subseteq \mathcal{B}$ with $\sum_{i \in \mathcal{D}} \alpha_{i}=1$ with $\alpha_{i}>0$ for any $i \in \mathcal{D}$. By Remark 1 , the set $\mathcal{D}$ uniquely includes abstention and extremal ballots. Furthermore, Lemma 1 entails that only extremal ballots belong to $\mathcal{D}$ as otherwise there is a contradiction with $\sigma$ being an equilibrium.

Formula (1) implies that, given $\sigma$, for any $\varepsilon>0$, there exists a positive pivot probability vector $p^{\varepsilon}$ such that

$$
E_{t}\left[c \mid p^{\varepsilon}\right]=E_{t}\left[\sum_{i \in \mathcal{D}} \alpha_{i} \cdot d^{i} \mid p^{\varepsilon}\right]=\sum_{i \in \mathcal{D}} \alpha_{i} \cdot E_{t}\left[d^{i} \mid p^{\varepsilon}\right] .
$$

In other words, given $p^{\varepsilon}$, the $t$-voter is indifferent between the strategy $\sigma(\cdot \mid t)$ (i.e. voting $c$ ) and the "mimicking" strategy $\gamma_{c}(\cdot \mid t)$ with $\gamma_{c}\left(d^{i} \mid t\right)=\alpha_{i}$ for all $d^{i} \in \mathcal{D}$.

We have proven so far that a $t$-voter is indifferent between casting ballot $c$ (as in $\sigma$ ) or playing the mixed strategy $\gamma_{c}(\cdot \mid t)$ that uniquely involves extremal ballots. Hence, given $\sigma(\cdot \mid-t)$, we have proven that $\gamma_{c}(\cdot \mid t)$ is a best response for $t$-voters. Note that as $c$ is a convex combination of ballots $d^{i}$ in some subset $\mathcal{D} \subseteq \mathcal{B}$, each $d^{i} \in \mathcal{D}$ is in $\operatorname{BR}_{t}\left(p^{\varepsilon}\right)$ as otherwise there is a contradiction with $\sigma$ being an equilibrium.

Denote by $\Gamma=\left\{\gamma_{c}(\cdot \mid t), \sigma(\cdot \mid-t)\right\}$ a strategy in which types $t^{\prime} \neq t$ play according to $\sigma$. In order to prove that $\Gamma$ is an equilibrium, we need to prove that any $t^{\prime}$-voter with $t^{\prime} \neq t$ is playing a best response. However, as the scores of the candidates are unchanged when $t$-voters switch from playing strategy $\sigma(\cdot \mid t)$ to strategy $\gamma_{c}(\cdot \mid t)$, the same pivot probability vector $p^{\varepsilon}$ justifies both strategy combinations. Therefore, the set of best responses of $t^{\prime}$-voters $\mathrm{BR}_{t^{\prime}}\left(p^{\varepsilon}\right)$ is unchanged as it only hinges on the pivot probability vector. Therefore, any $t^{\prime}$-voter is playing a best response in the strategy combination $\Gamma$, proving that $\Gamma$ is an equilibrium

If type $t$ is playing a mixed strategy $\sigma(\cdot \mid t)$, it suffices to apply the above argument to each interior ballot $c$ played with positive probability and to take as the mixed strategy mimicking $\sigma(\cdot \mid t)$ the average of $\gamma_{c}(\cdot \mid t)$, according to $\sigma$.

All in all, both $\sigma$ and $\Gamma$ are justified by the same pivot probability vector and under both of them, the expected scores of the candidates coincide. Hence, for any 
equilibrium $\sigma$ in which the $t$-voters cast an interior ballot, there exists an equivalent equilibrium $\Gamma$ in which they only cast extremal ballots.

As the argument does not hinge neither on the type $t$ nor on the strategies played by the rest of the voters, one can repeat the same argument in equilibrium $\gamma$ if some voter plays an interior ballot with positive probability. Hence, by iteratively applying this argument, one can prove that for any interior equilibrium, there exists an equivalent extremal one.

\subsection{A Sufficient Condition for Strategic equivalence}

Proposition 1 proves that interior equilibria are not informative in the sense that they do not add any information regarding the equilibria that can be attained under a voting rule. Building on such a result, we now give a simple sufficient condition to ensure the strategic equivalence of two voting rules.

Adding the same constant to every candidate in a ballot obviously does not change anything, so we normalize rules in the following way. Let $U$ be a voting rule with ballot set $\mathcal{B}_{U}$. Let $N: \mathcal{B} \rightarrow \mathcal{B}$ the normalizing transformation which brings to 0 the minimal grade in any ballot: $(N(b))_{j}=b_{j}-\min _{i \in K} b_{i}$ for all $j$. Denote by $N(U)$ the normalized version of $U$ with ballot set $N\left(\mathcal{B}_{U}\right)$. We omit the proof of the following result:

Proposition 2. Any voting rule $U$ is strategically equivalent to its normalized version $N(U)$.

From now on, we concentrate on normalized rules. As will be shown, the strategic equivalence of two rules depends on their extremal ballots. Recall that a homothetic transformation is a transformation $H: X \rightarrow Y$ of the form $H(x)=s+t x$ is with both $s, t$ real numbers, $t>0$. Two vectors $x$ and $y$ are homothetic if there exists a homothetic transformation between them. Similarly, two sets are homothetic if every pair of vectors in $X$ and $Y$ are homothetic. A dilation is a homothetic transformation with $s=0$.

Theorem 1. If there exists a homothetic transformation between the sets of extremal ballots of two normalized voting rules $U$ and $V$, then $U$ and $V$ are strategically equivalent.

Proof. Let $U$ and $V$ denote two voting rules such that there exists a transformation $f: b \longmapsto f(b)=\alpha+\beta b$, from $\operatorname{Ext}\left(\mathcal{B}_{U}\right)$ onto $\operatorname{Ext}\left(\mathcal{B}_{V}\right)$, for some reals $\alpha, \beta$ with 
$\beta>0$. As both $U$ and $V$ are normalized, we know that every ballot $b \in \mathcal{B}_{U} \cup \mathcal{B}_{V}$, has positive coordinates, the smallest one being 0 . Hence, $\alpha=0$.

Let $\sigma_{U}$ be a strategy in an election held under $U$ in which every voter only casts ballots in the set $\operatorname{Ext}\left(\mathcal{B}_{U}\right)$. Let $\sigma_{V}$ denote a strategy in the same election held under $V$ that satisfies

$$
\sigma_{V}\left(b^{*} \mid t\right)=\sigma_{U}(b \mid t), \quad \forall t \in \mathcal{T},
$$

in which each ballot $b^{*}$ satisfies $b^{*}=f(b)=\beta b$. Hence, in the strategy combination $\sigma_{V}$, every voter only casts ballots in the $\operatorname{set} \operatorname{Ext}\left(\mathcal{B}_{V}\right)$.

The scores of the candidates $S_{U}(\cdot)$ given $\sigma_{U}$ and $S_{V}(\cdot)$ given $\sigma_{V}$ satisfy

$$
S_{V}\left(c_{i}\right)=\beta S_{U}\left(c_{i}\right) \forall c_{i} \in \mathcal{K},
$$

and hence the scores of candidates coincide up to an homothetic transformation under both strategies.

Let us now prove that if $\sigma_{U}$ is an equilibrium then the strategy $\sigma_{V}$ is an equilibrium with $\sigma_{U}$ and $\sigma_{V}$ being equivalent.

As $\sigma_{U}$ is an equilibrium, there exists a vector $p^{\varepsilon}=\left(p_{i j}^{\varepsilon}\right)_{i j \in H}$ with $p_{i j}^{\varepsilon}>0$ for every $i j \in H$ and every $\varepsilon>0$ that satisfies the ordering condition for every $\varepsilon>0$. Furthermore, $p^{\varepsilon}$ justifies $\sigma_{U}$. As under both $\sigma_{U}$ and $\sigma_{V}$ the relative scores of the candidates coincide, the same vector $p^{\varepsilon}$ satisfies the ordering condition for both strategies for any $\varepsilon>0$. Hence, it remains to be proven that $p^{\varepsilon}$ justifies the strategy $\sigma_{V}$

Therefore, we have that, for every $\varepsilon>0, p^{\varepsilon}$ justifies the strategy $\sigma_{U}$, so that for every $b \in \operatorname{Supp}_{t}\left(\sigma_{U}\right)$ :

$$
\begin{aligned}
b \in \arg \max _{d \in \operatorname{Ext}\left(\mathcal{B}_{U}\right)} E_{t}\left[d \mid p^{\varepsilon}\right] & \Longleftrightarrow E_{t}\left[b \mid p^{\varepsilon}\right] \geq E_{t}\left[d \mid p^{\varepsilon}\right] \forall d \in \operatorname{Ext}\left(\mathcal{B}_{U}\right) \\
& \Longleftrightarrow \beta E_{t}\left[b \mid p^{\varepsilon}\right] \geq \beta E_{t}\left[d \mid p^{\varepsilon}\right] \forall d \in \operatorname{Ext}\left(\mathcal{B}_{U}\right) \\
& \Longleftrightarrow E_{t}\left[b^{*} \mid p^{\varepsilon}\right] \geq E_{t}\left[d^{*} \mid p^{\varepsilon}\right] \forall d^{*} \in \operatorname{Ext}\left(\mathcal{B}_{V}\right) \\
& \Longleftrightarrow b^{*} \in \arg \max _{d^{*} \in \operatorname{Ext}\left(\mathcal{B}_{V}\right)} E_{t}\left[d^{*} \mid p^{\varepsilon}\right] .
\end{aligned}
$$

We have therefore proven that if $b \in \operatorname{Supp}_{t}\left(\sigma_{U}\right)$ when the election is held under $U$, then $b^{*}=\beta_{U V} b \in \operatorname{BR}_{t}\left(p^{\varepsilon}\right)$ when the election is held under $V$.

We can now conclude that $\sigma_{V}$ is an equilibrium. Indeed, as the scores under both $\sigma_{U}$ and $\sigma_{V}$ coincide up to a homothetic transformation, the pivot probability vector $p^{\varepsilon}$ satisfies the ordering condition for strategy $\sigma_{V}$ for any $\varepsilon>0$. Furthermore, by definition, $\sigma_{U}\left(b^{*} \mid t\right)>0$. Additionally, as $b \in \operatorname{Supp}_{t}\left(\sigma_{U}\right)$, then $b^{*} \in \operatorname{BR}_{t}\left(p^{\varepsilon}\right)$. Therefore, $\sigma_{V}$ is an equilibrium when the election is held under $V$. 
As the $f$ function is bijective, it can be proven similarly that if $\sigma_{V}$ is an equilibrium under $V$, then $\sigma_{U}$ is an equilibrium under $U$. In other words, if there exists an homothetic transformation between $\operatorname{Ext}\left(\mathcal{B}_{U}\right)$ and $\operatorname{Ext}\left(\mathcal{B}_{V}\right)$, the set of extremal equilibria under both $U$ and $V$ are equivalent. But the previous equivalence finishes the proof as, by Proposition 1, any interior equilibrium under a voting rule is equivalent to an extremal equilibrium under the same voting rule.

Theorem 1 has the advantage of being extremely simple to use: indeed, as will be shown by section 5, almost no computation is needed to check the strategic equivalence of two voting rules.

\subsection{A Necessary Condition for the Strategic Equivalence of Rank Scoring Rules}

A natural question to ask is whether the previous sufficient condition for strategic equivalence is also necessary. We only have a partial answer to this technically challenging question. We prove that the condition is necessary if we restrict the analysis to rank scoring rules.

Theorem 2. Let $U$ and $V$ be two rank scoring rules. If $U$ and $V$ are strategically equivalent, then there exists a homothetic transformation between their sets of extremal ballots.

First notice that two rank scoring rules are homothetic if and only if they are identical once re-scaled between 0 and 1 . Within the proof, we uniquely consider re-scaled rank scoring rules w.l.o.g. due to Theorem 1. The proof of the theorem is presented in the next paragraphs. We start by the case of three candidates and then generalize the argument.

Three candidates. Take a scoring rule $U$ with weights $\left(1, s_{2}, 0\right)$. Let the pivot vector $p^{\varepsilon}=\left(p_{i j}^{\varepsilon}\right)_{i j \in H}$ satisfy $p_{12}^{\varepsilon}=p_{13}^{\varepsilon}=\varepsilon$ and $p_{23}^{\varepsilon}=\varepsilon^{2}$.

Suppose that there are three possible types of voters in the election: the ones who prefer $c_{1}$ to $c_{2}$ and $c_{2}$ to $c_{3}$, the ones who prefer $c_{3}$ to $c_{1}$ and $c_{1}$ to $c_{2}$ and finally the ones who prefer $c_{2}$ to $c_{3}$ and $c_{3}$ to $c_{1}$. All voters assign a utility of 10 to their first candidate, a utility of 5 to their middle candidate and no utility to their least

preferred one. We denote by $\beta_{i}$ the share of voters who rank candidate $c_{1}$ in the $i^{t} h$ position; it follows that $\beta_{3}=1-\beta_{1}-\beta_{2}$. Given the pivot probabilities, one can 
easily check that the voters' best responses are sincere so that they assign a higher weight to a more preferred candidate than to a less preferred one.

Given these best responses, the scores of the candidates are the following ones:

$$
\begin{aligned}
& S\left(c_{1}\right)=\beta_{1}+\beta_{2} s_{2} ; \\
& S\left(c_{2}\right)=\beta_{1} s_{2}+\left(1-\beta_{1}-\beta_{2}\right) ; \\
& S\left(c_{3}\right)=\beta_{2}+\left(1-\beta_{1}-\beta_{2}\right) s_{2} .
\end{aligned}
$$

Letting $\beta_{2}^{*}=\beta_{1}^{*} \frac{2 s_{2}-1}{2-s_{2}}+\frac{1-s_{2}}{2-s_{2}}$ and $\beta_{1}^{*}>1 / 3$ leads to $S\left(c_{1}\right)>S\left(c_{2}\right)=S\left(c_{3}\right)$ which is compatible with the ordering of pivot of probabilities. In other words, given the type distribution $\beta^{*}$ and the pivot probabilities vector, there exists an equilibrium under the voting rule $U$. Take now another rank scoring rule $V$ with weights $\left(1, s_{2}^{\prime}, 0\right)$ and w.l.o.g. assume that $s_{2}^{\prime}>s_{2}$. Given the type distribution is $\beta^{*}$ and the pivot vector, the best responses are identical as in $V$ so that the score vector $S^{\prime}$ satisfies

$$
\begin{aligned}
& S^{\prime}\left(c_{2}\right)=\beta_{1}^{*} s_{2}^{\prime}+\left(1-\beta_{1}^{*}-\beta_{2}^{*}\right) ; \\
& S^{\prime}\left(c_{3}\right)=\beta_{2}^{*}+\left(1-\beta_{1}^{*}-\beta_{2}^{*}\right) s_{2}^{\prime} .
\end{aligned}
$$

As $\beta_{1}^{*}>1 / 3$, one can check that $\left(1-\beta_{1}^{*}-\beta_{2}^{*}\right)<\beta_{1}^{*}$. Hence, $\beta_{1}^{*} s_{2}^{\prime}>\left(1-\beta_{1}^{*}-\beta_{2}^{*}\right) s_{2}^{\prime}$ so that $S^{\prime}\left(c_{2}\right)>S^{\prime}\left(c_{3}\right)$. These scores are in contradiction with $p_{12}^{\varepsilon}=p_{13}^{\varepsilon}=\varepsilon$ entailing that there is not a corresponding equilibrium when the election is held under $V$.

Therefore, we have proven the lack of strategic equivalence of rank scoring rules for three candidates. We now prove the lack of strategic equivalence for any number of candidates.

Multi-candidate elections. The proof proceeds as follows. We first construct for any rank scoring rule $U$ a type distribution and an equilibrium such that all candidates but one get the same expected score. We then deduce from the previous point that given the type distribution, this equilibrium does not exist under any different rank scoring rule $V$. Therefore, the rules $U$ and $V$ are not strategically equivalent.

Before proving the main claim, we introduce a lemma dealing with voters' best responses that will be useful throughout.

Say that a scoring ballot is sincere if it matches the voter's ordinal preference:

Definition 6. A scoring rule ballot $c$ is sincere for a t-voter if

$$
u_{t}\left(c_{i}\right)>u_{t}\left(c_{j}\right) \Longrightarrow s_{i} \geq s_{j}
$$


Observe that under a rank scoring rule, if a voter has strict preferences, he has a unique sincere ballot. The next lemma proves that, when all pivots are equally likely, this sincere ballot is his unique best response.

Lemma 2. Assume that a voter has strict preferences over the candidates. If all pivot probabilities are equal in an election held under a rank scoring rule, then the unique best response for the voters is sincere.

Proof. Assume that all pivot probabilities are equal, i.e. $p_{i j}^{\varepsilon}=\varepsilon$ for every $i j \in$ $H$. Pick a scoring rule with weights $\left(s_{i}\right)_{i=1}^{k}$ and a voter with preferences $u_{t}\left(c_{1}\right)>$ $u_{t}\left(c_{2}\right)>\ldots>u_{t}\left(c_{k}\right)$. Hence, the sincere ballot for the $t$-voter is the ballot $b^{*}=$ $\left(1, s_{2}, s_{3}, \ldots, s_{k-1}, 0\right)$. Let us now prove that the unique best response for $t$-voters is ballot $b^{*}$. Take two integers $m$ and $n$ with $m<n$ and denote by $\rho^{m n}$ the vector in $\mathbb{R}^{k}$ that assigns 1 point to coordinate $m,-1$ point to coordinate $n$ and 0 to the rest of coordinates. Due to (1), we can write that

$$
E_{t}\left[\rho^{m n} \mid p^{\varepsilon}\right]=\sum_{i j \in H}\left(\rho_{i}^{m n}-\rho_{j}^{m n}\right) \cdot p_{i j} \cdot\left[u_{t}\left(c_{i}\right)-u_{t}\left(c_{j}\right)\right] .
$$

Note that as $p_{i j}^{\varepsilon}=\varepsilon$ for every $i j \in H$, we can remove the pivot probabilities as they do not affect the sign of the expected utility so that $E_{t}\left[\rho^{m n} \mid p^{\varepsilon}\right]=\sum_{i j \in H}\left(\rho_{i}^{m n}-\right.$ $\left.\rho_{j}^{m n}\right) \cdot\left[u_{t}\left(c_{i}\right)-u_{t}\left(c_{j}\right)\right]$. As $\rho_{m}^{m n}=1, \rho_{n}^{m n}=-1$ and $\rho_{j}^{m n}=0$ for any $j \neq m, n$, it follows that

$$
E_{t}\left[\rho^{m n} \mid p^{\varepsilon}\right]=k\left[u_{t}\left(c_{m}\right)-u_{t}\left(c_{n}\right)\right] .
$$

Denote by $\mu_{l}$ the ballot that assigns 1 point to candidate $l$ and no points to the rest of the candidates. Using the previous equality, we can set up a complete preference order over the different ballots $\mu_{l}$ for the $t$-voters. Indeed, as by definition, $u_{t}\left(c_{1}\right)>$ $u_{t}\left(c_{2}\right)>\ldots>u_{t}\left(c_{k}\right)$, it follows that

$$
E_{t}\left[\mu_{1} \mid p^{\varepsilon}\right]>E_{t}\left[\mu_{2} \mid p^{\varepsilon}\right]>\ldots>E_{t}\left[\mu_{k} \mid p^{\varepsilon}\right]
$$

Therefore, the $t$-voters' best response is the one that assigns the highest weight to candidate 1 , the second highest weight to candidate 2 , and so on which actually coincides with the definition of sincere ballot under a scoring rule, concluding the proof.

Equipped with this lemma, we continue with the proof of Theorem 2 for multicandidate elections. Assume that the voters anticipate that all pivots in which candidate $c_{1}$ is not involved are equally likely and that they are infinitely less likely 
than the ones involving candidate $c_{1}$. Formally, assume that the pivot probabilities are ordered as follows:

$$
p_{1 j}^{\varepsilon}=\varepsilon \text { if } j \neq 1 \text { and } p_{i j}^{\varepsilon}=\varepsilon^{2} \text { for every } i, j \neq 1 \text {. }
$$

Let the voters' preferences be strict and the number of types be equal to $k$, the number of candidates. We denote each type by $t_{i}$ in which $i$ stands for the rank assigned by voters to candidate $c_{1}$. Moreover, all voters rank candidate $c_{j+1}$ immediately after candidate $c_{j}$ if possible.

In other words, the cardinal preferences of a $t_{i}$-voter, $1 \leq i \leq k$, satisfy

$$
u_{t_{i}}\left(c_{k-i+2}\right)>\ldots>u_{t_{i}}\left(c_{k}\right)>u_{t_{i}}\left(c_{1}\right)>u_{t_{i}}\left(c_{2}\right)>\ldots>u_{t_{i}}\left(c_{k-i+1}\right),
$$

in which $c_{i}=c_{i-k}$ if $i>k$.

Given the order of the probabilities, the unique best response for both $t_{1}$ and $t_{k}$-voters is to vote sincerely. Indeed, following Lemma 2, voters' best responses are sincere if all pivot probabilities are equal. Even though it is not the case here, sincerity follows from candidate $c_{1}$ being either the first or the worst choice for all these voters combined with the fact that all pivot probabilities in which $c_{1}$ is not involved are equal. It hence follows that these voters assign 1 point to $c_{1}$. Moreover, the weights assigned to the rest of the candidates hinge only on the pivots in which $c_{1}$ is involved (the rest of the pivots being infinitely less likely) and, as they are all equal, the logic of Lemma 2 applies.

As far as the other voters are concerned, the logic of Lemma 2 does not apply directly, and one needs to specify some utility levels to ensure that the unique best response is sincere. Indeed, a $t_{i}$-voter with $t_{i} \neq t_{1}, t_{k}$ has candidate $c_{k-i+2}$ as his most preferred alternative, $c_{1}$ in the $i^{t} h$ position and $c_{k-i+1}$ as his least preferred alternative. As for the case of $t_{1}$ and $t_{k}$ voters, their best response is sincere if we restrict the analysis to the weights assigned to all the candidates but $c_{1}$. Indeed, as all the pivot probabilities in which $c_{1}$ is involved are equal, the logic of Lemma 2 applies. However, whether it is in their interest to be sincere when considering also the weight assigned to $c_{1}$ depends on their cardinal utility.

As previously defined, $\rho^{1 n}$ stands for the vector in $\mathbb{R}^{k}$ that assigns 1 point to coordinate $1,-1$ point to coordinate $n$ and 0 to the rest of the coordinates. In order to ensure that their unique best response is sincere, it must be the case that for any $t_{i}$-voter with $t_{i} \neq t_{1}, t_{k}$, there exists an $\hat{\varepsilon}>0$ such that for any $\varepsilon$ in $(0, \hat{\varepsilon})$, the following inequalities hold:

$$
E_{t_{i}}\left[\rho^{1 n} \mid p^{\varepsilon}\right]>0 \text { for } n \in\{2, \ldots, k-i+1\}
$$


and

$$
E_{t_{i}}\left[\rho^{1 n} \mid p^{\varepsilon}\right]<0 \text { for } n \in\{k-i+2, \ldots, k\} .
$$

Given the sequence of pivot probabilities (3), one can check that

$$
\lim _{\varepsilon \rightarrow 0} \frac{E_{t_{i}}\left[\rho^{1 n} \mid p^{\varepsilon}\right]}{\varepsilon}=k u_{t_{i}}\left(c_{1}\right)-u_{t_{i}}\left(c_{n}\right)-\sum_{j=2}^{k} u_{t_{i}}\left(c_{j}\right) .
$$

Hence, in order to ensure that the unique best response for $\varepsilon$ small is sincere, it suffices to assume that

$$
\frac{u_{t_{i}}\left(c_{2}\right)+\sum_{j=2}^{k} u_{t_{i}}\left(c_{j}\right)}{k}<u_{t_{i}}\left(c_{1}\right)<\frac{u_{t_{i}}\left(c_{k}\right)+\sum_{j=2}^{k} u_{t_{i}}\left(c_{j}\right)}{k},
$$

as $c_{k}$ is ranked immediately above $c_{1}$ and $c_{2}$ is ranked immediately below. We therefore assume that the previous inequality holds for every $t_{i}$-voter with $t_{i} \neq t_{1}, t_{k}$.

Let $\beta_{i}$ denote the share of $t_{i}$-voters who rank candidate $c_{1}$ on the $i^{t} h$ position. Note that the numbers $\beta_{i}$ can be chosen independently the ones from the others with the constraint that their sum equals one.

Take a rank scoring rule $U$ with weights $\left(s_{i}\right)_{i=1}^{k}$. As the rank scoring rule is re-scaled, $s_{1}=1$ and $s_{k}=0$. Assume that the pivot probabilities are ordered as in (3) so that voters' best responses are sincere.

Let $\hat{s}$ stand for the extension of the voting weights $\left(s_{i}\right)_{i=1}^{k}$ for any positive integer $i$ as follows :

$$
\hat{s}_{i}= \begin{cases}s_{i} & \text { if } 1 \leq i \leq k \\ s_{i}=s_{i-k} & \text { if } i>k .\end{cases}
$$

Given the best responses, the score vector $S$ is as follows:

$$
\begin{aligned}
S\left(c_{1}\right) & =\sum_{i=1}^{k} \beta_{i} \hat{s}_{i} ; \\
S\left(c_{2}\right) & =\sum_{i=1}^{k} \beta_{i} \hat{s}_{i+1} ; \\
\ldots & =\ldots \\
S\left(c_{j}\right) & =\sum_{i=1}^{k} \beta_{i} \hat{s}_{i+j-1} ; \\
\ldots & =\cdots \\
S\left(c_{k}\right) & =\sum_{i=1}^{k} \beta_{i} \hat{s}_{i+k-1} .
\end{aligned}
$$


The sequence of pivot probabilities (3) is only compatible with the scores of candidates being ordered as $S\left(c_{1}\right) \geq S\left(c_{2}\right)=\ldots=S\left(c_{k}\right)$.

Given the previous system of equations, we can write that $S\left(c_{j}\right)=S\left(c_{j+1}\right)$ for any $j \geq 2$ if and only if

$$
\sum_{i=1}^{k} \beta_{i}\left(\hat{s}_{i+j-1}-\hat{s}_{i+j}\right)=0 .
$$

Moreover, $S\left(c_{1}\right) \geq S\left(c_{2}\right)$ holds when $\sum_{i=1}^{k} \beta_{i}\left(\hat{s}_{i}-\hat{s}_{i+1}\right) \geq 0$.

Note that the equality among all candidates holds when $\beta_{i}=\frac{1}{k}$ for every $i$ (a Condorcet cycle exists under such a distribution). Therefore, as there are $k-1$ variables $\left(\beta_{i}\right.$ for $i=1, \ldots, k-1$ as $\left.\sum_{i} \beta_{i}=1\right), k-2$ equations and one inequality, there exists a continuum of solutions. For the rest of type distributions for which the equilibrium is ensured, the type distribution satisfies $\beta_{1}>1 / k$ which moreover implies that :

$$
\beta_{1}>\beta_{i} \text { for any } i \neq 1 \text {, }
$$

when some $s_{i} \neq 1$.

Now, take two different scoring rules $U$ and $V$ with associated weights $\left(s_{i}\right)_{i=1}^{k}$ and $\left(s_{i}^{\prime}\right)_{i=1}^{k}$. Take a type distribution $\beta^{*}$ under which the previously described equilibrium under $U$ exists. We shall prove that the equilibrium under $U$ corresponds to no equilibrium under $V$, and hence that both rules are not strategically equivalent. The proof is by contradiction and is divided in three different cases labeled $\mathbf{a}, \mathbf{b} \mathbf{1}$, b2.

a. Suppose first that they differ by one term, say $s_{l} \neq s_{l}^{\prime}$ and $s_{j}=s_{j}^{\prime}$ for any $j \neq l$. Consider the type distribution $\beta^{*}$ and the sequence of pivot probabilities (3). The best responses are identical under both $U$ and $V$. Note that the $t_{1}$-voters assign $s_{l}$ points to $c_{l}$. If we denote by $S^{\prime}$ the score vector under $V$, we have

$$
S^{\prime}\left(c_{l}\right)-S\left(c_{l}\right)=\beta_{1}^{*}\left(s_{l}^{\prime}-s_{l}\right),
$$

and for any $c_{i} \neq c_{l}$,

$$
S^{\prime}\left(c_{j}\right)-S\left(c_{j}\right)=\beta_{i_{0}}^{*}\left(s_{l}^{\prime}-s_{l}\right),
$$

with $i_{0}$ such that $i_{o}+j-1=l$. As by construction $\beta_{1}^{*}>\beta_{j}^{*}$ for any $j \neq 1$, it follows that $S^{\prime}\left(c_{l}\right)-S\left(c_{l}\right)>S^{\prime}\left(c_{j}\right)-S\left(c_{j}\right)$ for any $c_{j} \neq c_{1}$. Hence, candidate $c_{l}$ has the second highest score, in contradiction with the sequence of pivot probabilities (3).

b. Suppose now that the weights of $U$ and $V$ differ by more than one term, say $s_{i} \neq s_{i}^{\prime}$ for any $i \in I \subseteq \mathcal{K}$ and $s_{j}=s_{j}^{\prime}$ for any $j \notin I$. 
b.1 Suppose first that the cardinal of $I$ equals 2. W.l.o.g. take $I=\left\{c_{2}, c_{3}\right\}$. With the type distribution $\beta^{*}$ and the sequence of pivot probabilities (3), the best responses are identical under both $U$ and $V$. Hence, we can write that

$$
S^{\prime}\left(c_{2}\right)-S\left(c_{2}\right)=\beta_{1}^{*}\left(s_{2}^{\prime}-s_{2}\right)+\beta_{2}^{*}\left(s_{3}^{\prime}-s_{3}\right)
$$

and

$$
S^{\prime}\left(c_{3}\right)-S\left(c_{3}\right)=\beta_{1}^{*}\left(s_{3}^{\prime}-s_{3}\right)+\beta_{k}^{*}\left(s_{2}^{\prime}-s_{2}\right) .
$$

Moreover, if there is an equilibrium under $V$ equivalent to the one described under $U$, it must be the case that $S^{\prime}\left(c_{2}\right)-S\left(c_{2}\right)=S^{\prime}\left(c_{3}\right)-S\left(c_{3}\right)$. However, this equality is equivalent to:

$$
\beta_{1}^{*}\left(s_{2}^{\prime}-s_{2}+s_{3}-s_{3}^{\prime}\right)=\beta_{k}^{*}\left(s_{2}^{\prime}-s_{2}\right)+\beta_{2}^{*}\left(s_{3}-s_{3}^{\prime}\right) .
$$

However, as $\beta_{1}^{*}>\beta_{i}^{*}$ for any $i \neq 1$, it follows that:

$$
\beta_{k}^{*}\left(s_{2}^{\prime}-s_{2}\right)+\beta_{2}^{*}\left(s_{3}-s_{3}^{\prime}\right)<\beta_{1}^{*}\left(s_{2}^{\prime}-s_{2}\right)+\beta_{1}^{*}\left(s_{3}-s_{3}^{\prime}\right)=\beta_{1}^{*}\left(s_{2}^{\prime}-s_{2}+s_{3}-s_{3}^{\prime}\right),
$$

which contradicts (4).

b.2 Suppose finally that the cardinal of $I$ equals 3 , the rest of the cases being analogous. W.l.o.g. take $I=\left\{c_{2}, c_{3}, c_{4}\right\}$. With the type distribution $\beta^{*}$ and the sequence of pivot probabilities (3), the best responses are identical under both $U$ and $V$. Hence, we can write that

$$
S^{\prime}\left(c_{2}\right)-S\left(c_{2}\right)=\beta_{1}^{*}\left(s_{2}^{\prime}-s_{2}\right)+\beta_{2}^{*}\left(s_{3}^{\prime}-s_{3}\right)+\beta_{3}^{*}\left(s_{4}^{\prime}-s_{4}\right),
$$

and

$$
S^{\prime}\left(c_{3}\right)-S\left(c_{3}\right)=\beta_{1}^{*}\left(s_{3}^{\prime}-s_{3}\right)+\beta_{2}^{*}\left(s_{4}^{\prime}-s_{4}\right)+\beta_{k}^{*}\left(s_{2}^{\prime}-s_{2}\right) .
$$

If there is an equilibrium under $V$ equivalent to the one described under $U$, we must that $S^{\prime}\left(c_{2}\right)-S\left(c_{2}\right)=S^{\prime}\left(c_{3}\right)-S\left(c_{3}\right)$. However, this equality implies that:

$$
\beta_{1}^{*}\left(s_{2}^{\prime}-s_{2}+s_{3}-s_{3}^{\prime}\right)=\beta_{2}^{*}\left(s_{3}-s_{3}^{\prime}\right)+\beta_{k}^{*}\left(s_{2}^{\prime}-s_{2}\right)+\beta_{3}^{*}\left(s_{4}-s_{4}^{\prime}\right)+\beta_{2}^{*}\left(s_{4}^{\prime}-s_{4}\right) .
$$

As $\beta_{1}^{*}>\beta_{i}^{*}$ for any $i \neq 1$, the right-hand side of (5) is strictly lower than

$$
\beta_{1}^{*}\left(s_{3}-s_{3}^{\prime}\right)+\beta_{1}^{*}\left(s_{2}^{\prime}-s_{2}\right)+\beta_{1}^{*}\left(s_{4}-s_{4}^{\prime}\right)+\beta_{1}^{*}\left(s_{4}^{\prime}-s_{4}\right)<\beta_{1}^{*}\left(s_{2}^{\prime}-s_{2}+s_{3}-s_{3}^{\prime}\right),
$$

entailing a contradiction with (5).

We can therefore conclude that any two scoring rules with different weights lead to different equilibria given the same pivot probabilities vector and hence are not strategically equivalent. 


\section{Applications}

Two applications of Theorem 1 are now described. The main interest of such a theorem is that it allows to "simplify" voting rules, in which the term simplify has been coined by the recent literature on mechanism simplification ${ }^{6}$. In this literature, a mechanism is simplified by reducing the message space of the agents, while no new equilibria are created as a consequence of this reduction. When the number of voters becomes large enough, adding or removing interior ballots to a voting rule does not modify the set of voting equilibria. Our results hence prove that when the number of voters is large enough, many voting rules can be simplified.

\subsection{Evaluative Voting: One man, Many extended votes}

Under Evaluative Voting with $m$ points, a voter can assign up to $m$ points to each candidate, for some positive $m$. Its set of abstention ballots equals $\operatorname{Abs}\left(\mathcal{B}_{E V^{m}}\right)=$ $\{(0, \ldots, 0),(m, \ldots, m)\}$. Hence, as $A V$ is a particular case of $E V^{m}$, the set of abstention ballots of $A V$ equals $\operatorname{Abs}\left(\mathcal{B}_{A V}\right)=\{(0, \ldots, 0),(1, \ldots, 1)\}$.

Corollary 1. For any finite $m, E V^{m}$ and $A V$ are strategically equivalent.

Proof. Note that the set of extremal ballots of $A V$ is not modified by normalizing the rule as it satisfies

$$
\operatorname{Ext}\left(\mathcal{B}_{A V}\right)=\{0,1\}^{k} \backslash\{(0, \ldots, 0),(1, \ldots, 1)\} .
$$

The same claim applies to the extremal ballots of $E V^{m}$ as it equals

$$
\operatorname{Ext}\left(\mathcal{B}_{E V^{m}}\right)=\{0, m\}^{k} \backslash\{(0, \ldots, 0),(m, \ldots, m)\}
$$

so that, by Theorem 1 and Proposition $2, E V^{m}$ and $A V$ are strategically equivalent.

\subsection{Cumulative Voting: One man, One extended vote}

In an election held under Cumulative Voting with $m$ points, a voter can assign up to $m$ points to each candidate for some positive $m$ with the restriction that the sum of the points he can assign to each of the candidates is at most $m$. Hence, its set of abstention ballots equals:

$$
\operatorname{Abs}\left(\mathcal{B}_{C V^{m}}\right)=\left\{(0, \ldots, 0),(1,1, \ldots, 1), \ldots,\left(\left\lfloor\frac{m}{k}\right\rfloor, \ldots,\left\lfloor\frac{m}{k}\right\rfloor\right)\right\}
$$

\footnotetext{
${ }^{6}$ See Milgrom $(2009,2010)[19,20]$ and Perez-Richet (2011) [29].
} 
with $\lfloor x\rfloor$ being the largest integer lower than $x . P V$ is a particular case of $C V^{m}$ with $m=1$ and hence its set of abstention ballots equals $\operatorname{Abs}\left(\mathcal{B}_{P V}\right)=\{(0, \ldots, 0)\}$ as $\left\lfloor\frac{1}{k}\right\rfloor=0$ for any $k$.

Corollary 2. For any finite $m, C V^{m}$ and $P V$ are strategically equivalent.

Proof. Given the set of ballots under $P V$ and $C V^{m}$, one obtains that $\operatorname{Ext}\left(\mathcal{B}_{P V}\right)$ consists of all the permutations of $(1,0, \ldots, 0)\}$ and that $\operatorname{Ext}\left(\mathcal{B}_{C V^{m}}\right)$ consists of all the permutations of $(1,0, \ldots, 0)\}$. Since the set of extremal ballots is not affected by normalization, Theorem 1 , concludes the proof.

Even though we have proven that $C V^{m}$ and $P V$ are strategically equivalent, we have remained silent over the set of possible winners in an election. To do so, we give a proposition which extends a previous result of De Sinopoli (2000) [8] (which focused in Plurality Voting with perfect equilibrium à la Selten) to the MyersonWeber setting. We show that any candidate who is not a Condorcet loser can win the election under Cumulative Voting and hence under Plurality voting.

Prior to stating it, we need the definition of Condorcet loser. For any pair of candidates $c_{i}, c_{j} \in \mathcal{K}$, let $V(i, j)=\left\{t \in \mathcal{T} \mid u_{t}\left(c_{i}\right)>u_{t}\left(c_{j}\right)\right\}$ be the set of types where candidate $c_{i}$ is strictly preferred to candidate $c_{j}$. The Condorcet Loser of the election is defined as:

Definition 7. A candidate $c_{i}$ is called the Condorcet Loser of the election if

$$
\sum_{t \in V(i, j)} r(t)<1 / 2 \forall c_{j} \in \mathcal{K}, c_{j} \neq c_{i}
$$

Proposition 3. Assume that voters have strict preferences. For every positive m, in an election held under $C V^{m}$, for every candidate $c_{i}$ who is not a Condorcet loser there exists an equilibrium in which $c_{i}$ wins the election with positive probability.

We provide a proof for completeness. The idea of the proof is standard and identical to the proofs for $P V$ in similar settings (De Sinopoli (2000) [8] and Myerson (2002) [23]).

Proof. Let $c_{1}$ and $c_{2}$ be two candidates. As voters have strict preferences, we divide the voters in two groups: the voters who prefer candidate $c_{1}$ to candidate $c_{2}, V(1,2)=\left\{t \in \mathcal{T} \mid u_{t}\left(c_{1}\right)>u_{t}\left(c_{2}\right)\right\}$, and the remaining ones $V(2,1)=\{t \in$ $\left.\mathcal{T} \mid u_{t}\left(c_{2}\right)>u_{t}\left(c_{1}\right)\right\}$. We suppose that $\sum_{t \in V(1,2)} r(t)>\sum_{t \in V(2,1)} r(t)$ (in case of an equality a similar claim applies). Under $C V^{m}$, a voter can assign up to $m$ points 
to a single candidate. Consider the strategy $\sigma(\cdot \mid t)$ such that every $t$-voter with $t \in V(1,2)$ assigns $m$ points to $c_{1}$, i.e.

$$
\sigma((m, 0, \ldots, 0) \mid t)=1
$$

and such that every $t^{\prime}$-voter with $t^{\prime} \in V(2,1)$ assigns $m$ points to $c_{2}$,

$$
\sigma\left((0, m, \ldots, 0) \mid t^{\prime}\right)=1
$$

Therefore, the winner of the election is candidate 1 given $\sigma$. This equilibrium is supported, for any $\varepsilon>0$, by any pivot probability vector $p^{\varepsilon}=\left(p_{i j}^{\varepsilon}\right)_{i j \in H}$ that satisfies two constraints. First, the limit when $\varepsilon$ tends towards zero of the conditional pivot probability vector $q^{\varepsilon}$ satisfies $q_{12}=1$ and $q_{i j}=0$ with $i j \neq 12$. Second, for any candidate $c_{l} \neq c_{1}, c_{2}$ and for any $\varepsilon>0, p_{2 l}^{\varepsilon} \leq \varepsilon p_{1 l}^{\varepsilon}$ in order to satisfy the ordering condition for $\varepsilon$. Given such a pivot probability vector, voters expect that, in the event of a pivot, the most probable pivot occurs candidates $c_{1}$ and $c_{2}$, the ones with the highest scores. Voters that prefer 1 to 2 assign the highest score to 1 and similarly for the voters that prefer $c_{2}$ to $c_{1}$. Indeed, take a $t$-voter with $t \in V(1,2)$. His expected utility gain equals $E_{t}\left[\left(b_{1}, b_{2}, \ldots, b_{k}\right) \mid p^{\varepsilon}\right]=\sum_{i j \in H}\left(b_{i}-b_{j}\right) \cdot p_{i j}^{\varepsilon} \cdot\left[u_{t}\left(c_{i}\right)-u_{t}\left(c_{j}\right)\right]$. As $q=(1,0, \ldots, 0)$, we can write that $E_{t}\left[\left(b_{1}, b_{2}, \ldots, b_{k}\right) \mid p\right]=\left(b_{1}-b_{2}\right)\left[u_{t}\left(c_{1}\right)-u_{t}\left(c_{2}\right)\right]$. Under $C V^{m}$, the ballot for which $b_{1}-b_{2}$ is maximized is $(m, 0, \ldots, 0)$. Hence, as in the rest of the ballots under $C V^{m}$, the difference $b_{1}-b_{2}$ is strictly lower than $m$, we have $\operatorname{BR}_{t}(p)=\{(m, 0, \ldots, 0)\}$ for a $t$-voter with $t \in V(1,2)$. A similar argument applies for $t^{\prime}$-voters with $t^{\prime} \in V(2,1)$, concluding the proof.

\section{Small Elections}

The results previously presented are a consequence of the model used in which voters' perceptions over the impact of their ballots in switching the winner of the election have a very specific shape. Such a theory fits particularly well the study of mass elections. Indeed, as shown by further developments of the theory ${ }^{7}$, more formal models give, roughly speaking, similar predictions depending on whether the ordering condition is satisfied. However, it seems that the specific shape of expected utility is not particularly relevant for studying voting in committees (that is voting with few voters). Indeed, in a committee, the information a voter knows can be much more detailed than in a large election.

\footnotetext{
${ }^{7}$ See Myerson (2002) [23], Laslier (2009) [16], Núñez (2009) [27], Bouton and Castanheira (2012) [4], Goertz and Maniquet (2011) [12], and Nuñez (2010) [26].
} 
In order to prove that non-extremal voting may be the unique best response in a voting game with few voters, we present an example. In order to test the robustness of the example, we focus on trembling-hand perfection à la Selten.

Let us recall that each voter chooses from a ballot set $\mathcal{B}$. Letting $\mathcal{B}^{n}$ stand for the product ballot set, we let $\Delta^{0}\left(\mathcal{B}^{n}\right)$ denote the set of all probability distributions over $\mathcal{B}^{n}$ which give positive probability to all members of $\mathcal{B}^{n}$. Hence a strategy profile $\sigma_{\mathcal{N}} \in \Delta^{0}\left(\mathcal{B}^{n}\right)$ is a completely mixed strategy profile in the game $(\mathcal{N}, \mathcal{B}, \mathcal{T}, \mathcal{K})$. The definition of perfection is as follows:

Definition 8. A completely mixed strategy profile $\sigma_{\mathcal{N}}^{\varepsilon}$ is an $\varepsilon$-perfect equilibrium in the game $(\mathcal{N}, \mathcal{B}, \mathcal{T}, \mathcal{K})$ if

$$
\forall i \in \mathcal{N}, \forall b^{i}, \bar{b}^{i} \in \mathcal{B} \text {, if } U_{i}\left(b^{i}, \sigma_{\mathcal{N} \backslash\{i\}}^{\varepsilon}\right)>U_{i}\left(\bar{b}^{i}, \sigma_{\mathcal{N} \backslash\{i\}}^{\varepsilon}\right) \text {, with } \sigma^{\varepsilon}\left(\bar{b}^{i}\right) \leq \varepsilon,
$$

in which $U_{i}(b)$ denotes the payoff of voter $i$ given the strategy combination $b . W e$ refer to the strategy combination $\sigma_{\mathcal{N}}$ as a perfect equilibrium if there exists a sequence $\left\{\sigma_{\mathcal{N}}^{\varepsilon}\right\}$ of $\varepsilon$-perfect equilibria converging (for $\varepsilon \rightarrow 0$ ) to $\sigma_{\mathcal{N}}$.

\section{Example 2.}

There are three candidates $\mathcal{K}=\left\{c_{1}, c_{2}, c_{3}\right\}$ and four different types $\mathcal{T}=\{\mathrm{a}, \mathrm{b}, \mathrm{c}, \mathrm{d}\}$, with cardinal utilities given by:

$$
u_{\mathrm{a}}=(6,1,0), \quad u_{\mathrm{b}}=(0,6,1), u_{\mathrm{c}}=(0,1,6) \text { and } u_{\mathrm{d}}=(0,3,6) .
$$

There are seven voters in the electorate. Voters 1,2 and $\mathbf{3}$ have type a, voters $\mathbf{4}$ and 5 have type b, voter 6 has type $\mathrm{c}$ and voter $\mathbf{7}$ has type $\mathrm{d}$.

We consider $E V^{2}$, that is Evaluative Voting in which voters can give up to two points to each of the candidates.

We let $g$ denote the strategy combination

$$
g=\left((2,0,0),(2,0,0),(2,0,0),(0,2,1),(0,2,1),(0,0,2), g_{7}\right)
$$

in which $g_{7}$ stands for the mixed strategy $1 / 3(0,0,2)+1 / 3(0,1,2)+(1 / 3)(0,2,2)$ of voter 7 . Every voter plays an undominated strategy in the strategy combination $g$. It is easy to check that $g$ is a mixed-strategy equilibrium of the election in which voters 1 to 6 are playing a unique best response.

Proposition 4. In Example 2, $g$ is a perfect equilibrium in which some voters' unique best responses are not extremal when the election is held under $E V^{2}$. 
Remark 3. Formula (1) is not verified in a perfect equilibrium in small elections.

The source of the non extremal behavior in this example is clearly the uncertainty faced by voters 4 and $\mathbf{5}$, as a consequence of the mixing of voter $\mathbf{7}$. The same logic applies in a (Bayesian) game of incomplete information in which voters are not sure of the type of their opponents.

Remark 4. The utility vectors are consistent with single-peaked preferences.

\section{Conclusion}

Building on the theory of strategic voting in large elections, we have derived sufficient and necessary conditions for the strategic equivalence of additive voting rules that simply depend on the ballots available to the voters. The sufficient condition says that whenever two voting rules share the same set of extremal ballots (up to a homothetic transformation), then they are strategically equivalent. This condition is proven to be necessary for the strategic equivalence of rank scoring rules.

Furthermore, the sufficient condition helps us to draw some conclusions about how adding ballots to a given voting rule modifies the set of voting equilibria. We set up a distinction between extensions of voting rules: completions (adding interior ballots) and enrichments (adding at least one extremal ballot). Whereas all voting rules are robust to completion, it might be the case than an enrichment modifies the set of equilibria of a voting rule. Using this distinction, we prove that it is possible to add ballots to both Plurality Voting and Approval Voting without modifying the set of voting equilibria. In the case of Approval Voting, there is no difference between $E V^{m}$ (for any $m$ ) and $A V$ when voters act strategically. As far as $P V$ is concerned, Cumulative Voting $\left(C V^{m}\right.$ for any $\left.m\right)$ extends $P V$ but remains strategically equivalent to $P V$. Nevertheless, the possibilities of expression seem to be somehow bounded for the following reason. Consider a voting rule which allows at most $M$ points. If one wishes to enlarge the possibilities of expression of voters by adding new ballots while respecting the same constraint, one can at best propose $E V^{M}$. But, the rule $E V^{M}$ is strategically equivalent to $E V^{1}$, which is $A V$. As has been shown, the previous results do not extend to a context with a reduced number of voters.

We have very few observations to back up, or to invalidate, these theoretical results. Laslier and Van der Straeten (2004) [15] report on an experiment comparing 
$E V$ with the 0 to 10 scale and $A V$, and Baujard and Igersheim (2010) [2] and Baujard et al. (2010) [1] report on experiments comparing the use of $E V$ under various scales, including $A V$. It is observed that the outcome of the election (the elected candidate) tends to be the same under different systems, even if it is not observed that voters concentrate on extreme grades.

An interesting extension of the present work would be to understand whether similar results apply under proportional representation or in multi-seat elections in which voters have to distribute their votes.

\section{A Appendix: Proof of Proposition 4}

The first step of the proof consists in showing that $g$ is a mixed strategy equilibrium. To do so, we compute the probability, under $g$, of each pivot outcome a player can face and, from these probabilities, the expected utility derived from each undominated strategy.

\section{Voters $1,2,3$}

Even though the best responses are explained for the voter 1, the reasoning is analogous for voters $\mathbf{2}$ and $\mathbf{3}$.

$p\left((4,4,6) \mid g_{-1}\right)=1 / 3$

$p\left((4,5,6) \mid g_{-1}\right)=1 / 3$

$p\left((4,6,6) \mid g_{-1}\right)=1 / 3$.

From the pivot probabilities previously described, we have

$U_{\mathbf{1}}(2,0,0)=25 / 9$

$U_{\mathbf{1}}(2,1,0)=19 / 9$

$U_{\mathbf{1}}(2,2,0)=13 / 9$.

which entails that $(2,0,0)$ is the unique best response for voter 1 .

\section{Voters 4,5}

Voter 4's best responses are analyzed, the reasoning being analogous for the voter 5 .

$p\left((6,2,5) \mid g_{-4}\right)=1 / 3$

$p\left((6,3,5) \mid g_{-4}\right)=1 / 3$

$p\left((6,4,5) \mid g_{-4}\right)=1 / 3$.

From the pivot probabilities previously described, we have

$U_{4}(0,2,0)=1$ 
$U_{4}(0,2,1)=10 / 9$

$U_{4}(0,2,2)=1$.

implying that $(0,2,1)$ is the unique best response for voter 4 .

\section{Voter 6}

The most probable pivot outcomes faced by voter $\mathbf{6}$ are as follows

$p\left((6,4,4) \mid g_{-6}\right)=1 / 3$

$p\left((6,5,4) \mid g_{-6}\right)=1 / 3$

$p\left((6,6,4) \mid g_{-6}\right)=1 / 3$

From the pivot probabilities previously described, we have

$U_{\mathbf{6}}(0,0,2)=25 / 9$

$U_{\mathbf{6}}(0,1,2)=19 / 9$

$U_{\mathbf{6}}(0,2,2)=13 / 9$.

implying that $(0,0,2)$ is the unique best response for voter 6 .

\section{Voter 7}

The most probable pivot outcome faced by voter 7 is the event $(6,4,4)$. Due to her utility profile, voter $\mathbf{7}$ strictly prefers to use an undominated strategy and is indifferent among all of them: that is $(0,0,2),(0,1,2),(0,2,2)$. Hence, the mixed strategy $g_{7}$ is a best response.

The second step of the proof consists in showing that $g$ is a perfect equilibrium. To do so, consider the following completely mixed strategy combination $g^{\varepsilon}$, where $\eta_{i}$ denotes the mixed strategy of voter $i$ which assigns equal probability to all his pure strategies.

$$
\begin{aligned}
& i=\mathbf{1}, \mathbf{2}, \mathbf{3} \quad g_{i}^{\varepsilon}=\left(1-27 \varepsilon^{2}\right)(2,0,0)+27 \varepsilon^{2} \eta_{i} \\
& i=\mathbf{4}, \mathbf{5} g_{i}^{\varepsilon}=\left(1-27 \varepsilon^{2}\right)(0,2,1)+27 \varepsilon^{2} \eta_{i} \\
& i=\mathbf{6} \quad g_{i}^{\varepsilon}=\left(1-\varepsilon-27 \varepsilon^{2}\right)(0,0,2)+\varepsilon(0,1,2)+27 \varepsilon^{2} \eta_{i}, \\
& i=\mathbf{7} \quad g_{i}^{\varepsilon}=g_{7}+27 \varepsilon^{2} \eta_{i} .
\end{aligned}
$$

It is easy to see that, for $\varepsilon$ sufficiently close to zero, this is an $\varepsilon$-perfect equilibrium. Suppose all voters other than $i$ choose the strategies prescribed by $g$. Since for $\varepsilon$ going to zero, the probability of voter 6 to tremble towards $(0,1,2)$ is infinitely greater than the probability of any other mistake, it is enough to check that the limiting strategy is preferred to the other undominated strategy when either this mistake or no mistake at all occurs. 
For voters $\mathbf{1}$ to $\mathbf{5}$, the relevant contingency is the one described by the limiting strategy $g$. Indeed, as has been shown, their unique best response is the one depicted by $g$ as when the trembles tends towards, they have a unique best response. For voter $\mathbf{6}$, the same argument applies.

Finally, one can deduce that for voter $i=7$ casting the mixed strategy ballot $g_{7}$ is a best response, against $g^{\varepsilon}$. Indeed, for voter 7 , the relevant contingency are summarized by the vectors $(6,4,4)$ and $(6,5,4)$. Let us denote their probabilities by $p\left((6,4,4) \mid f_{-i}^{\varepsilon}\right)$ and $p\left((6,6,4) \mid f_{-i}^{\varepsilon}\right)$. Since

$$
\begin{aligned}
U_{\mathbf{7}}(0,0,2) & =3 p\left((6,4,4) \mid f_{-i}^{\varepsilon}\right)+3 p\left((6,5,4) \mid f_{-i}^{\varepsilon}\right) \\
& =U_{\mathbf{7}}(0,1,2), U_{\mathbf{7}}(0,2,2) .
\end{aligned}
$$

the mixed strategy $g_{7}$ is a best reply to $g_{-i}^{\varepsilon}$.

Hence, $\left\{g^{\varepsilon}\right\}$ is a sequence of $\varepsilon$-perfect equilibria. Since $g$ is the limit of $g^{\varepsilon}$, it is a perfect equilibrium in which voters' best responses are not extremal.

\section{References}

[1] A. Baujard, F. Gavrel, H. Igersheim, J.-F. Laslier, and I. Lebon. Compte-rendu de l'expérimentation des votes par approbation et par note lors des élections Présidentielles Françaises le 22 avril 2012 à Saint-Etienne, Strasbourg et Louvigny. mimeo, GATE, 2012.

[2] A. Baujard and H. Igersheim. Framed Field Experiments on Approval Voting: lessons from the 2002 and 2007 French Presidential Elections. In J.F. Laslier and R. Sanver, editors, Handbook on Approval Voting. Heildelberg: SpringerVerlag, 2010.

[3] A. Blais, L. Massicotte, and A. Dobrzynska. Direct Presidential Elections: A World Summary. Electoral Studies, 16:441-455, 1997.

[4] L. Bouton and M. Castanheira. One Person, Many Votes: Divided Majority and Information Aggregation. Econometrica, 80:43-87, 2012.

[5] S. Brams. Game Theory and Politics. Free Press, New York, 1975.

[6] G.W. Cox. Centripetal and Centrifugal Incentives in Electoral Systems. American Journal of Political Science, 34:903-935, 1990. 
[7] C. d'Aspremont and L. Gevers. Social Welfare Functionals and Interpersonal Comparability. In K.J. Arrow, A.K. Sen, and K. Suzumura, editors, Handbook of Social Choice and Welfare. Amsterdam: Elsevier, 2002.

[8] F. De Sinopoli. Sophisticated Vote and Equilibrium Refinements under Plurality Rule. Social Choice and Welfare, 17:655-672, 2000.

[9] A. Dhillon and J-F. Mertens. Relative Utilitarianism. Econometrica, 67:471498, 1999.

[10] W. Gaertner and Y. Xu. A general Scoring Rule. Mathematical Social Sciences, 6:193-196, 2012.

[11] E. Gerber, R. Morton, and T. Rietz. Minority Representation in Multimember Districts. American Political Science Review, 92:127-144, 1998.

[12] J. Goertz and F. Maniquet. On the Informational Efficiency of Simple Scoring Rules. Journal of Economic Theory, 146:1464-1480, 2011.

[13] E. Karni. Impartiality: Definition and Representation. Econometrica, 66:14051415, 1998.

[14] J-F. Laslier. Interpretation of electoral mixed strategies. Social Choice and Welfare, 17:283-292, 2000.

[15] J-F. Laslier and K. Van der Straeten. Une expérience de vote par assentiment lors de l'élection présidentielle française de 2002. Revue française de science politique, 54:99-130, 2004.

[16] J.F. Laslier. Strategic Approval Voting in a Large Electorate. Journal of Theoretical Politics, 21:113-136, 2009.

[17] J.F. Laslier and R. Sanver. The Basic Approval Voting Game. In J.F. Laslier and R. Sanver, editors, Handbook on Approval Voting. Heildelberg: SpringerVerlag, 2010.

[18] R.D. McKelvey and J. Patty. A theory of Voting in Large Elections. Games and Economic Behavior, 57:155-180, 2006.

[19] P. Milgrom. Assignment Messages and Exchanges. American Economic Journal: Microeconomics, 1:95-113, 2009. 
[20] P. Milgrom. Simplified Mechanisms with an Application to Sponsored-search Auctions. Games and Economic Behavior, 70:62-70, 2010.

[21] R. Myerson. Theoretical Comparisons of Electoral Systems. European Economic Review, 43:671-697, 1999.

[22] R. Myerson. Large Poisson Games. Journal of Economic Theory, 94:7-45, 2000.

[23] R. Myerson. Comparison of Scoring Rules in Poisson Voting Games. Journal of Economic Theory, 103:219-251, 2002.

[24] R. Myerson and R.J. Weber. A Theory of Voting Equilibria. American Political Science Review, 87:102-114, 1993.

[25] S. Nitzan. The Vulnerability of Point-Voting Schemes to Preference Variation and Strategic Manipulation. Public Choice, 47:349-370, 1985.

[26] M. Núñez. Condorcet Consistency of Approval Voting: A Counter Example on Large Poisson Games. Journal of Theoretical Politics, 22:64-84, 2010.

[27] M. Núñez. The Strategic Sincerity of Approval Voting. THEMA 2010-02 Working papers, 2010.

[28] T. Palfrey. A Mathematical Proof of Duverger's Law. In P.C. Ordeshook, editor, Models of Strategic Choice in Politics. The University of Michigan Press, 1989.

[29] E. Perez-Richet. A Note on The Tight Simplification of Mechanisms. Economics Letters, 110:15-17, 2011.

[30] J. Sawyer and D. MacRae. Game Theory and Cumulative Voting in Illinois: 1902-1954. American Political Science Review, 56:936-946, 1962.

[31] U. Segal. Let's Agree that All Dictatorships are Equally Bad. Journal of Political Economy, 108:569-589, 2000.

[32] R. Selten. A Reexamination of the Perfectness Concept for Equilibrium Points in Extensive Games. International Journal of Game Theory, 4:25-55, 1975.

[33] J. Smith. Aggregation of Preferences with a Variable Electorate. Econometrica, 41:1027-1041, 1973.

[34] P. Young. Social Choice Scoring Functions. SIAM Journal of Applied Mathematics, 27:824-838, 1975. 